\title{
Au/Fe/Au multilayer transducers for magneto-optic surface plasmon resonance sensing
}

\author{
D. Regatos, ${ }^{1, a}{ }^{1}$ D. Fariña, ${ }^{1}$ A. Calle, ${ }^{2}$ A. Cebollada, ${ }^{2}$ B. Sepúlveda, ${ }^{1}$ G. Armelles,${ }^{2}$ and \\ L. M. Lechuga ${ }^{1}$ \\ ${ }^{1}$ Nanobiosensors and Bioanalytical Applications Group, Research Center on Nanoscience \\ and Nanotechnology (CIN2) CSIC-ICN \& CIBER-BBN, 08193 Bellaterra, Barcelona, Spain \\ ${ }^{2}$ Microelectronics Institute of Madrid (IMM) CSIC-CNM, 28760 Tres Cantos, Madrid, Spain
}

(Received 26 April 2010; accepted 10 July 2010; published online 3 September 2010)

\begin{abstract}
In this paper, we analyze the magnetoplasmonic (MP) features and sensing capabilities of $\mathrm{Au} / \mathrm{Fe} / \mathrm{Au}$ trilayer structures, as transducers of the magneto-optic surface plasmon resonance (MOSPR) biosensor. This biosensor, which can surpass the sensitivity of the standard SPR sensor, is based on a MP modulation technique generated by the simultaneous stimulation of the surface plasmon polaritons (SPP) and the transversal magneto-optical Kerr effect (TMOKE). We study the magneto-optical activity of the trilayers as a function of the thickness and position of the Fe layer. We first demonstrate that this kind of structure allows modulating the SPP through an external magnetic field and moreover, induce a strong enhancement of the TMOKE effect. The modulation of the SPP is linearly proportional to the thickness of Fe layer and inversely proportional to the distance between the Fe layer and the external dielectric medium. Finally, we experimentally confirm a twofold increase in the MOSPR sensitivity with respect to the intensity-interrogated SPR biosensor in bulk refractive-index changes, keeping a similar chemical resistance and stability, unprecedented in other MP transducers, and biofunctionalization protocols. () 2010 American Institute of Physics. [doi:10.1063/1.3475711]
\end{abstract}

\section{INTRODUCTION}

Surface plasmon polaritons (SPP) are collective oscillations of the free-electron gas density at the boundary of a metal and a dielectric, the first with negative and the second with positive dielectric constant. The collective oscillation generates an electromagnetic wave which propagates along the interface of both media and creates highly confined electromagnetic fields whose maximum intensity is located at the metal/dielectric interface and decays exponentially at both sides of this. ${ }^{1,2}$ The SPP wave vector $\left(\overrightarrow{\mathbf{k}}_{\text {spp }}=\overrightarrow{\mathbf{k}}_{\mathbf{0}} \times \mathbf{n}_{\text {metal }}\right.$ $\left.\times \mathbf{n}_{\mathbf{d}} / \sqrt{\mathbf{n}_{\text {metal }}^{2}+\mathbf{n}_{\mathbf{d}}^{2}}\right)$, and hence the SPP excitation condition, strongly depends on the refractive index of the external dielectric medium $\left(\mathrm{n}_{\mathrm{d}}\right)$. Such strong dependence has enabled the development of a family of biosensors based on this phenomenon. ${ }^{3}$ Nearly thirty years after the birth of the surface plasmon resonance (SPR) biosensor, this analytical technique has become an essential tool for the real-time and label-free analysis of biospecific interactions. ${ }^{4}$ Several SPR biosensing configurations can be distinguished, ${ }^{5}$ depending on the characteristics of the light interacting with the SPP that are evaluated (SPR with angular, wavelength, intensity, phase, or polarization interrogation). Even though the sensitivity of SPR sensors is highly dependent on the spectral position of the resonance, ${ }^{6}$ their performance is not linked to the employed excitation or interrogation technique. ${ }^{7}$ Therefore, due to its simplicity and low-cost, the intensityinterrogated SPR configuration is one of the most widely employed. Such configuration is based on measuring the intensity of the reflected p-polarized monochromatic light at a

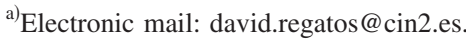

fixed angle of incidence. Although this type of configuration has shown a high sensitivity, such sensitivity could not be enough for the direct detection of low concentrations of small molecules. For this reason, surpassing the sensitivity of this particular configuration while keeping all its advantages would be an extremely valuable achievement. It is well known that modulation techniques can improve the signalto-noise ratio (SNR) and, therefore, enhance the limits of detection (LOD) in any kind of sensor. For this reason, in the last decade, different SPR modulation schemes have been proposed to improve the LOD of the SPR biosensor such as the mechanical ${ }^{8,9}$ and the phase ${ }^{10-12}$ modulated SPR sensors. In this context, a simple modulation alternative, based on the combination of magneto-optic effects and the SPR was recently proposed, denoted as the magneto-optic surface plasmon resonance (MOSPR $)^{13}$ biosensor. Such modulation technique has shown an experimental threefold increase in the LOD in refractive-index changes and in the adsorption of biomolecules with respect to conventional intensityinterrogated SPR sensors. Nevertheless, theoretical calculations estimate a LOD improvement of one order of magnitude for the MOSPR with respect to SPR sensors, if the setup and the metallic multilayer transducer are conveniently optimized.

The MOSPR biosensor is based on a magnetoplasmonic (MP) modulation technique produced in multilayers of noble and ferromagnetic metals (MP structure). These structures present plasmonic and magneto-optical (MO) activity, allowing the simultaneous excitation of SPP and the transversal magneto-optical Kerr effect $\left(\mathrm{TMOKE}^{14}\right)$ and generating a nonreciprocal shift in the $\mathrm{k}_{\mathrm{spp}}{ }^{15,16}$ The reversal of the mag- 

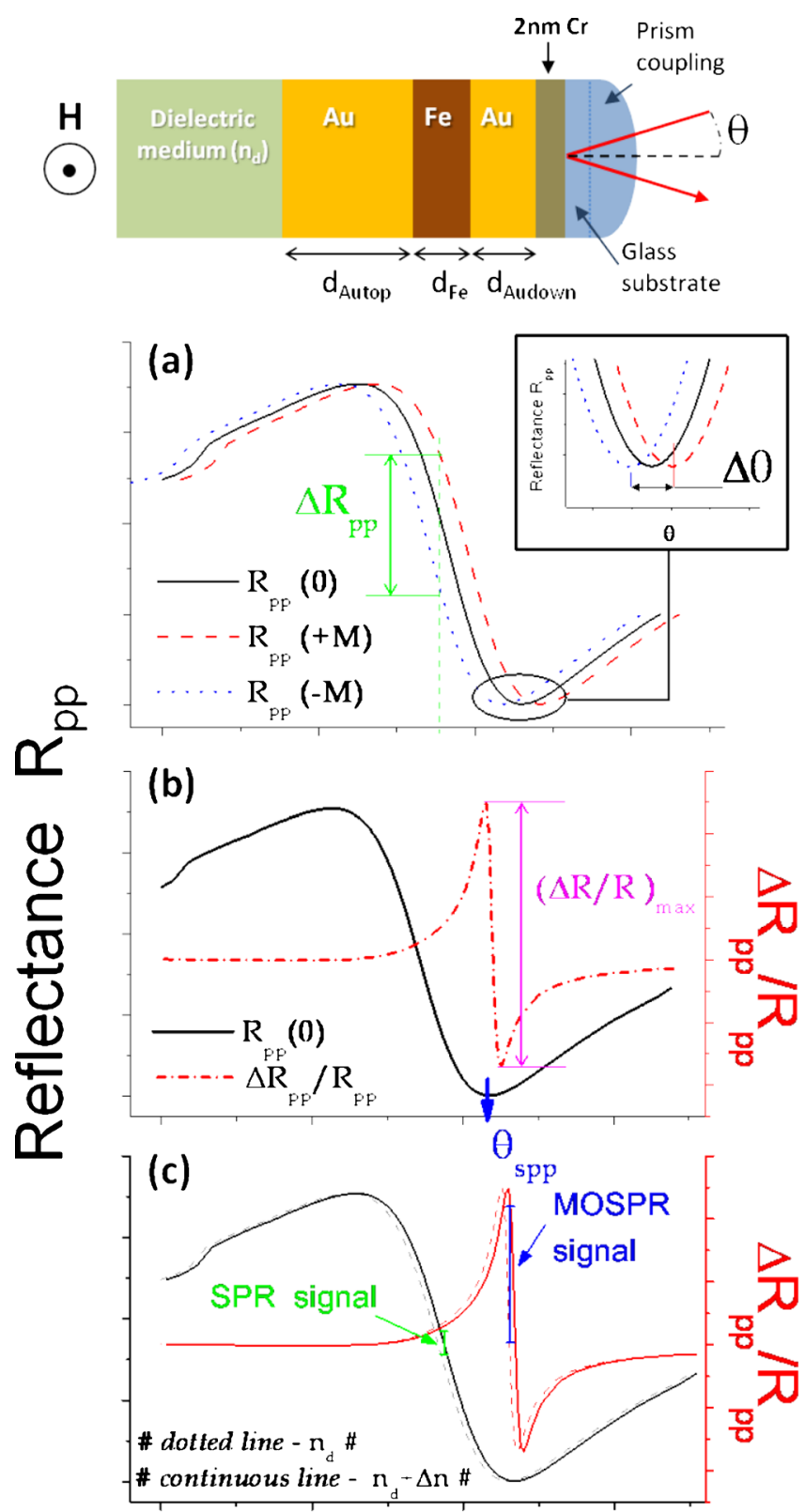

$\theta$

FIG. 1. (Color online) Top: schematic view of the employed MP trilayer transducer in transversal configuration. The SPP is excited through a coupling prism (Kretschmann configuration). Panels: (a) MO modulation of $\theta_{\text {spp }}$ by an alternating magnetic field. The inset shows the graph definition of the MO modulation factor $(\Delta \theta)$, (b) schematic plot of the enhancement of TMOKE effect produced when the SPP excitation condition is satisfied, and (c) schematic response of SPR and MOSPR sensors to the same variation in the external refractive index $(\Delta n)$.

netization $(\vec{M})$, produced by an external (ac) magnetic field, allows modulating the SPP excitation condition [see Fig. 1(a)]. The latter is translated into a relative variation in the reflected p-polarized light $\left(\mathrm{R}_{\mathrm{pp}}\right)$

$$
\frac{\Delta \mathrm{R}_{\mathrm{pp}}}{\mathrm{R}_{\mathrm{pp}}}=\frac{\mathrm{R}_{\mathrm{pp}}(\mathrm{M})-\mathrm{R}_{\mathrm{pp}}(-\mathrm{M})}{\mathrm{R}_{\mathrm{pp}}(0)},
$$

where $R_{p p}( \pm M)$ and $R_{p p}(0)$ are the reflectance of the p-polarized light with magnetization and in the demagnetized state, respectively.
The MO effects of a MP multilayer are strongly amplified when the SPP excitation condition is satisfied, ${ }^{17-19}$ which is reflected in a great enhancement of $\Delta R_{p p} / R_{p p}$ [see Fig. 1(b)] in resonance condition. Such enhancement strongly depends on the excitation conditions of the SPP and, therefore, on $\mathrm{n}_{\mathrm{d}}$, providing the sensing principle of the MOSPR device. Unlike intensity-interrogated SPR biosensor, which is based on the measurement of the $R_{p p}$, the MOSPR biosensor monitors the variations in the amplified $\Delta R_{p p} / R_{p p}$ induced by the excitation of SPP [see Fig. 1(c)]. Compared to the intensity-interrogated SPR sensor, the MOSPR sensor only requires an electromagnet placed in transversal configuration, and a MP transducer instead of the gold layer transducer of the SPR biosensor. These features turn the MOSPR biosensor into a simple solution to improve the LOD of SPR biosensors, which could be of paramount utility for high sensitivity applications, as for example in clinical diagnostics, ${ }^{20,21}$ food safety, ${ }^{22}$ and environmental protection. ${ }^{23}$

In order to surpass the sensing features of the SPR sensor, the MP transducer must have a large MO activity and a well-defined SPR; i.e., a sharp and angular narrow resonance. In a previous work, a $\mathrm{Au} / \mathrm{Cr} / \mathrm{Co}$ multilayer was employed as MOSPR transducer. ${ }^{13}$ The intermediate $\mathrm{Cr}$ layer was required to improve the stability of the transducer, due to the lack of adhesion between the Au and Co layers. Contrary to what would be desirable, this layer increases the optical absorption and reduces the MO effect of the multilayer, thus decreasing the sensitivity of the MP transducer. In this work, we explore the possibility of using $\mathrm{Fe}$ instead $\mathrm{Co}$ as ferromagnetic material of the MP transducer. Although $\mathrm{Fe}$ and $\mathrm{Co}$ have similar MO activity and optical absorption, ${ }^{24}$ the required magnetic field to saturate the Fe film is lower (20 Oe) than that for $\mathrm{Co}\left(50 \mathrm{Oe}^{13}\right)$, due to the lower magnetic anisotropy of $\mathrm{Fe}$, and the adhesion with $\mathrm{Au}$ is much better than for $\mathrm{Co}$, allowing the elimination of the intermediate $\mathrm{Cr}$ layer.

\section{EXPERIMENTAL PROCEDURE}

To evaluate the MP properties of the $\mathrm{Au} / \mathrm{Fe} / \mathrm{Au}$ transducer [see Fig. 1], we deposited two sets of $\mathrm{Au} / \mathrm{Fe} / \mathrm{Au}$ trilayers on glass substrates previously coated with $2 \mathrm{~nm}$ of $\mathrm{Cr}$ to improve the adherence. We focused our study on the influence of the position of the Fe layer with respect to the external medium, determined by the upper Au layer thickness $\left(\mathrm{d}_{\text {Autop }}\right)$, and the influence of the ferromagnetic layer thickness $\left(\mathrm{d}_{\mathrm{Fe}}\right)$ on the MP properties of the $\mathrm{Au} / \mathrm{Fe} / \mathrm{Au}$ structure. The trilayers were deposited by dc magnetron sputtering at room temperature in an ultrahigh-vacuum chamber with a base pressure of $10^{-9}$ mbar. For one set, the Fe layer thickness was $5 \mathrm{~nm}$, while for the other was kept at $3 \mathrm{~nm}$. Thin ferromagnetic layers were used to minimize the loss of sensitivity due to their optical absorption. For all samples, the thickness of the Au layer in contact with the glass substrate $\left(\mathrm{d}_{\text {Audown }}\right)$ was kept at $5 \mathrm{~nm}$. Therefore, we maintained the distance between the substrate and the ferromagnetic layer constant, only allowing $\mathrm{d}_{\text {Autop }}$ and $\mathrm{d}_{\mathrm{Fe}}$ to change. For both 
sets of trilayers $d_{\text {Autop }}$ was varied between 22 and $36 \mathrm{~nm}$, since the optimum theoretical value of the upper Au layer thickness is around $30 \mathrm{~nm}$.

In the MOSPR biosensor setup, the SPP was excited by prism-coupling (Krestchmann configuration ${ }^{25}$ ) and detected via angular interrogation [see Fig. 1]. The light source was a p-polarized $\mathrm{He}-\mathrm{Ne}$ laser, and the reflected light was collected with a Si photodiode. The TMOKE was generated through an electromagnet placed in transversal configuration, which changes the magnetization of the Fe layer between its two saturation states $\left( \pm \mathrm{M}_{\mathrm{sat}}\right)$ with a magnetic field of $30 \mathrm{Oe}$ and a frequency of $18 \mathrm{~Hz}$. The modulated signal was processed by a lock-in amplifier. A flow system composed of a flow cell, a flow-injection valve, and a peristaltic pump allowed easily interchanging the refraction index of the liquid external medium.

\section{RESULTS AND DISCUSSION}

As previously mentioned, the MO variation in the reflectance $\left(\Delta \mathrm{R}_{\mathrm{pp}}\right)$ in the transversal configuration is a consequence of the nonreciprocal change in $\mathrm{k}_{\mathrm{spp}}$. In the particular case of incident monochromatic light, such $\mathrm{k}_{\mathrm{spp}}$ variation is translated into a shift in the excitation angle $\theta_{\text {spp }}$ of the SPP [see Fig. 1(a)]. As a consequence, $\Delta \mathrm{R}_{\mathrm{pp}}$ can be approximated by $^{16}$

$$
\Delta \mathrm{R}_{\mathrm{pp}}=\frac{\partial \mathrm{R}_{\mathrm{pp}}}{\partial \theta} \times \Delta \theta
$$

where $\Delta \theta$ is defined as the angular shift in $\theta_{\text {spp }}$, in absolute value [see inset of Fig. 1(a)], when the magnetization is reversed, i.e., $\Delta \theta=\left|\theta_{\text {spp }}(+\mathrm{M})-\theta_{\text {spp }}(-\mathrm{M})\right|$. Therefore, we first analyzed the $\mathrm{MO}$ properties of the $\mathrm{Au} / \mathrm{Fe} / \mathrm{Au}$ structures through the dependence of the MO modulation factor $(\Delta \theta)$ on $\mathrm{d}_{\text {Autop }}$ and $\mathrm{d}_{\mathrm{Fe}}$. The experimental $\Delta \theta$ was obtained as the ratio between the maximums of the experimental $\Delta \mathrm{R}_{\mathrm{pp}}$ curves [see Fig. 2(a)] and their corresponding reflectance angular derivative $\left(\partial \mathrm{R}_{\mathrm{pp}} / \partial \theta\right)$, as can be deduced from Eq. (2). Figure 2(b) shows that, for a constant thickness of $\mathrm{d}_{\mathrm{Fe}}, \Delta \theta$ is reduced as the thickness of the upper Au layer increases. In addition, $\Delta \theta$ is larger for thicker Fe layers and the increase is proportional to $\mathrm{d}_{\mathrm{Fe}}$. Such MO behavior is related to the electromagnetic field distribution of the SPP inside the metal, in particular, due to its exponential decay inside the multilayer. Therefore, the closer the ferromagnetic layer is located to the metal-dielectric interface; the higher the electromagnetic field intensity inside this layer, therefore generating a higher MO modulation factor.

Once the effect of the position and thickness of the Fe layer is understood, its influence on the TMOKE, which is the actual magnitude measured for biosensing applications, can be studied as a function of both parameters. Figure 3 shows the angular $\Delta \mathrm{R}_{\mathrm{pp}} / \mathrm{R}_{\mathrm{pp}}$ curves of both sets of trilayers. For a constant Fe thickness [see Fig. 4(a)], the enhancement of the TMOKE effect produced in resonance condition [defined in Fig. 1(b) as $\left.(\Delta R / R)_{\max }\right]$ is achieved for a specific $\mathrm{d}_{\text {Autop }}$, different to that maximizing the MO modulation factor $\Delta \theta$. The optimum thickness of the upper Au layer was 32 $\mathrm{nm}$ and $30 \mathrm{~nm}$ for the multilayers with $3 \mathrm{~nm}$ and $5 \mathrm{~nm}$ of $\mathrm{Fe}$, (a)

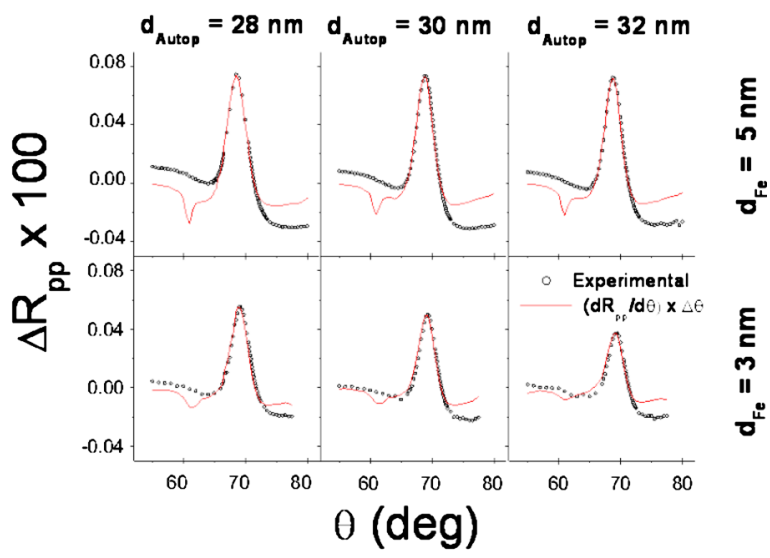

(b)

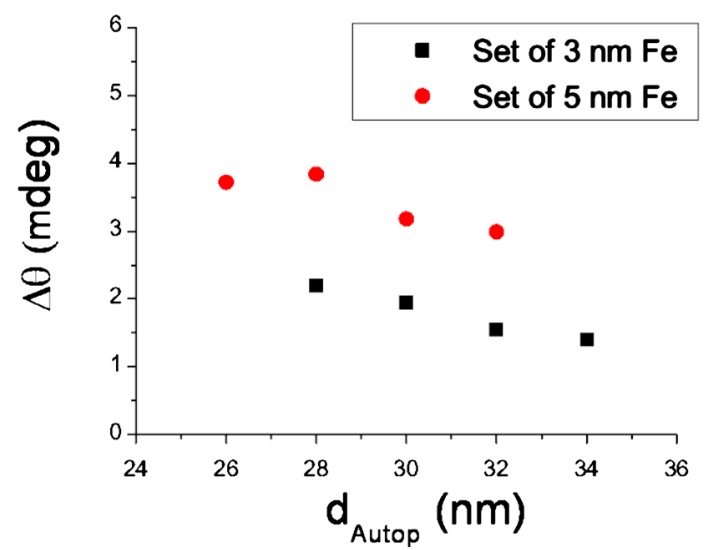

FIG. 2. (Color online) (a) Experimental MO variation in the reflectance $\left(\Delta \mathrm{R}_{\mathrm{pp}}\right)$ for three representative samples of both sets and angular derivative of their corresponding experimental $\mathrm{R}_{\mathrm{pp}}$ multiplied by their experimental $\Delta \theta$. (b) Experimental MO modulation factor $(\Delta \theta)$ as a function of the upper Au layer thickness.

respectively. The reason of such difference is because the SPP excitation condition of each set is optimized for a particular thickness of the multilayer, in which the $\mathrm{R}_{\mathrm{pp}}$ value at $\theta=\theta_{\text {spp }}\left(\mathrm{R}_{\text {min }}\right)$ is minimized [see Fig. 4(b)]. Under these conditions, the angular $\Delta \mathrm{R}_{\mathrm{pp}} / \mathrm{R}_{\mathrm{pp}}$ curve exhibits the maximum slope [see Fig. 4(c)] and, therefore, the angular curve for biosensing applications is also maximized.

By comparing structures with the same $\mathrm{d}_{\text {Autop }}$ and different $d_{\mathrm{Fe}}$ [see Fig. 4(d)], we observed that the Fe thickness determines the width of the $R_{p p}$ angular curve. A larger amount of $\mathrm{Fe}$ induces a larger optical absorption in the multilayer, which broadens the $\mathrm{R}_{\mathrm{pp}}$ angular curve, and hence the $\Delta \mathrm{R}_{\mathrm{pp}} / \mathrm{R}_{\mathrm{pp}}$ angular curve. On the other hand, the amount of Fe controls the MO activity of the multilayer, and therefore the amplitude of $\Delta \theta$. Equation (2) clearly points out that the choice of the optimum Fe layer thickness must be a compromise between the absorption of the ferromagnetic film [to maximize $\left.\left(\partial \mathrm{R}_{\mathrm{pp}} / \partial \theta\right)\right]$ and its MO activity (to amplify $\Delta \theta$ ). Inspection of Fig. 4 evidences that the increase in the MO effect for the thicker Fe films compensates the broadening of the reflectance curve, thus providing steeper and more intense $\Delta R_{p p} / R_{p p}$ curves, matching the requirement to improve the performance for sensing applications. As a consequence, we selected the multilayer composed of $30 \mathrm{~nm} \mathrm{Au}$ top $/ 5 \mathrm{~nm}$ $\mathrm{Fe} / 5 \mathrm{~nm} \mathrm{Au}$ down $_{1} / 2 \mathrm{~nm} \mathrm{Cr} /$ glass as MP sensing transducer as it exhibits the highest detection slope, and we compared it to 

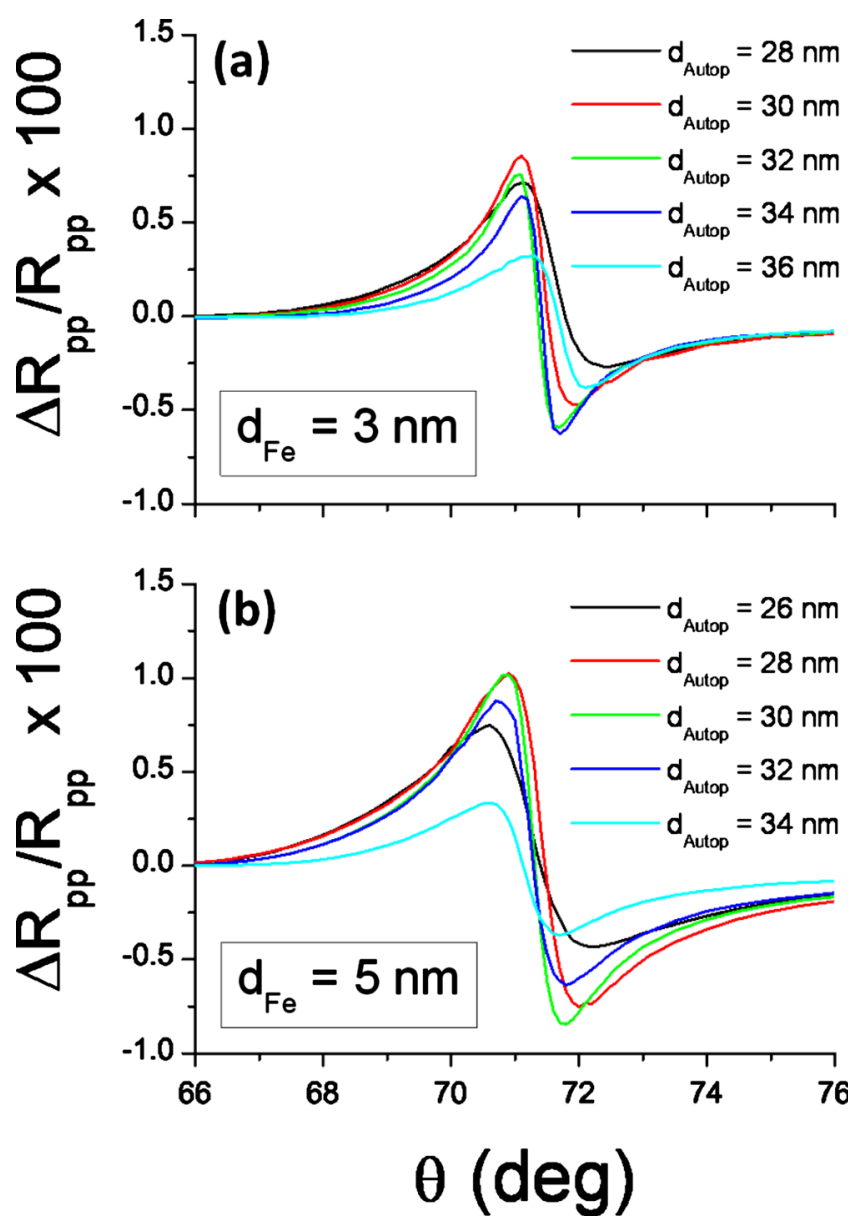

FIG. 3. (Color online) Experimental angular curves of $\Delta R_{p p} / R_{p p}$ for (a) 3 $\mathrm{nm} \mathrm{Fe}$ set $\left(\mathrm{d}_{\text {Autop }} / 3 \mathrm{~nm} \mathrm{Fe} / 5 \mathrm{nmAu} / 2 \mathrm{~nm} \mathrm{Cr} /\right.$ glass $)$ and (b) $5 \mathrm{~nm} \mathrm{Fe} \mathrm{set}$ $\left(\mathrm{d}_{\text {Autop }} / 5 \mathrm{~nm} \mathrm{Fe} / 5 \mathrm{nmAu} / 2 \mathrm{~nm} \mathrm{Cr} /\right.$ glass $)$.

the performance of a conventional SPR transducer. As SPR transducer, we used the optimal configuration at a wavelength of $632 \mathrm{~nm}$, given by $48 \mathrm{~nm} \mathrm{Au} / 2 \mathrm{~nm} \mathrm{Cr} /$ glass. Both structures were deposited on the same kind of substrate, using the same growth technique and conditions. The evaluation of the two sensors was done in the same experimental setup and flowing the same solutions of different refractive indices. Due to the different nature of the MO measurements of the MOSPR sensor and the reflectance measurements of the conventional SPR sensor, the comparison of their sensitivity must be done taking into account the SNR of the experimental measurements. We defined the system noise as the RMS deviation of the experimental signal (during $1000 \mathrm{~s})$, keeping $\mathrm{n}_{\mathrm{d}}$ constant. The changes in refractive index $(\Delta \mathrm{n})$ were generated flowing aqueous solutions with different $\mathrm{HCl}$ concentration (from 1 to $50 \mathrm{mM}$ ), equivalent to refractive-index changes from $1 \times 10^{-5}$ to $8 \times 10^{-4}$ refractive index units (RIU). The real-time detection of the $n_{d}$ variations were done at the angle that maximizes the slope of the $\Delta \mathrm{R}_{\mathrm{pp}} / \mathrm{R}_{\mathrm{pp}}$ or $\mathrm{R}_{\mathrm{pp}}$ curves for MOSPR and SPR sensors, respectively [see Fig. 1(c)].

The experimental results yield a twofold enhancement of the MOSPR sensor sensitivity to changes in $\mathrm{n}_{\mathrm{d}}$ as compared to the intensity-interrogated SPR sensor, using the $\mathrm{Au} / \mathrm{Fe} / \mathrm{Au}$ trilayer structure as transducer [see Fig. 5(a)]. This sensitiv-
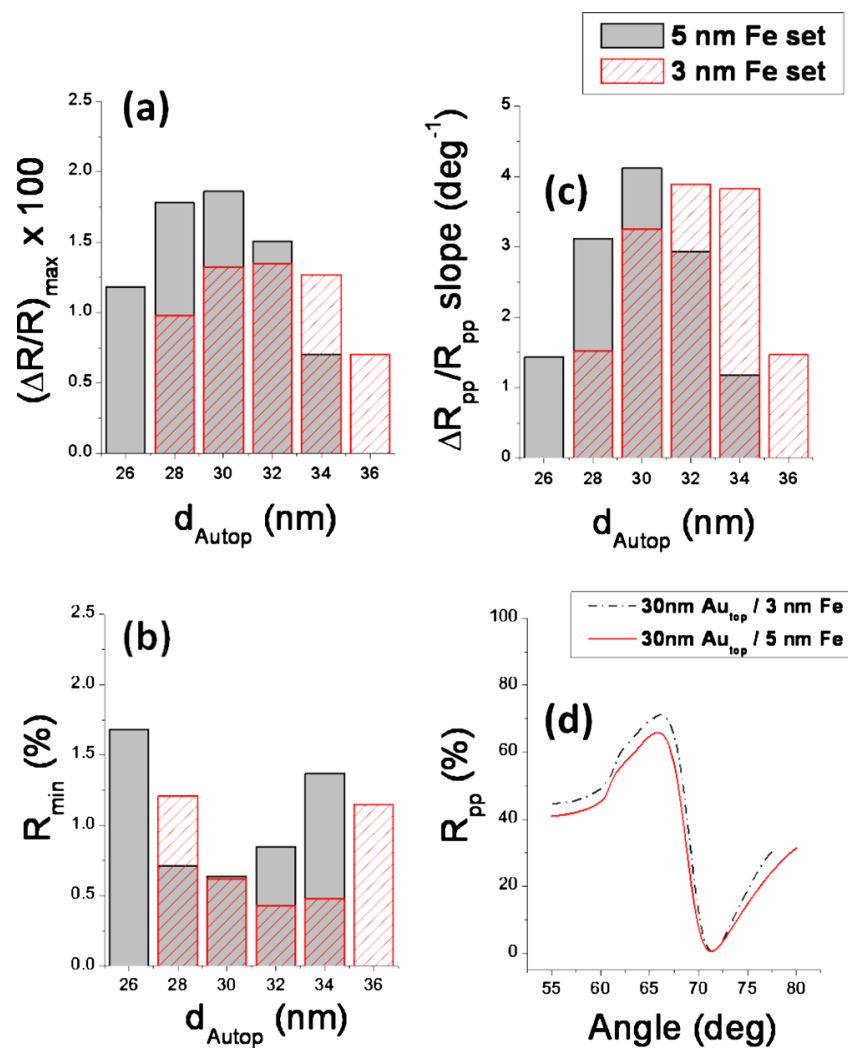

FIG. 4. (Color online) For all the samples grown and as a function of the thickness of Au upper layer: (a) experimental TMOKE increase produced in SPP resonance condition, (b) $\mathrm{R}_{\mathrm{pp}}\left(\theta=\theta_{\text {spp }}\right)$ value, and (c) the maximum value of the angular derivative of $\Delta \mathrm{R}_{\mathrm{pp}} / \mathrm{R}_{\mathrm{pp}}$. (d) Experimental angular $\mathrm{R}_{\mathrm{pp}}$ curve for the $(30 \mathrm{~nm} \mathrm{Au}$ top $/ 5 \mathrm{~nm} \mathrm{Fe} / 5 \mathrm{~nm} \mathrm{Au}$ down $/ 2 \mathrm{~nm} \mathrm{Cr} / g$ lass $)$ and $(30 \mathrm{~nm}$ $\mathrm{Au}_{\text {top }} / 3 \mathrm{~nm} \mathrm{Fe} / 5 \mathrm{~nm} \mathrm{Au}$ down $/ 2 \mathrm{~nm} \mathrm{Cr} /$ glass) structures.

ity increase, however, is still lower than that achieved by $\mathrm{Au} / \mathrm{Cr} / \mathrm{Co}$ multilayer structures, ${ }^{13}$ and far from the expected theoretical maximum sensitivity increase (of one order of magnitude). This is directly originated by the low values experimentally observed $\Delta \mathrm{R}_{\mathrm{pp}}$ in the $\mathrm{Au} / \mathrm{Fe} / \mathrm{Au}$ system, ten times lower than those predicted in theoretical calculations [see Fig. 5(b)]. Such drastic attenuation of $\Delta \mathrm{R}_{\mathrm{pp}}$ is likely due to the sizeable interface roughness in the $\mathrm{Au} / \mathrm{Fe} / \mathrm{Au}$ system, which gives rise to a region where $\mathrm{Fe}$ and $\mathrm{Au}$ coexist. This region can be viewed as an intermediate layer whose MO properties differ from those of pure $\mathrm{Fe}$, giving rise to a reduced MO activity, ${ }^{26}$ which in turn decreases the MO activity with respect to the ideal $\mathrm{Au} / \mathrm{Fe} / \mathrm{Au}$ trilayer. Since the experimental MO modulation factor is linearly proportional to the Fe layer thickness, this may explain the reduced MO activity observed with respect to the theoretically expected values. Exploring alternatives to fabricate $\mathrm{Au} / \mathrm{Fe} / \mathrm{Au}$ structures with sharper interfaces would probably be a route to obtain MO activity values closer to the theoretically expected ones.

On the other hand, as an advantage of the present system, we have observed that it provides a very good chemical resistance and stability to the MP transducer. Preliminary biosensing tests have proved that $\mathrm{Au} / \mathrm{Fe} / \mathrm{Au}$ trilayers were stable in solutions with very low $\mathrm{pH}$ or high ionic strength, which has not been achieved so far in Au/Co MP transducers. In addition, thiol-based immobilization protocols could be 

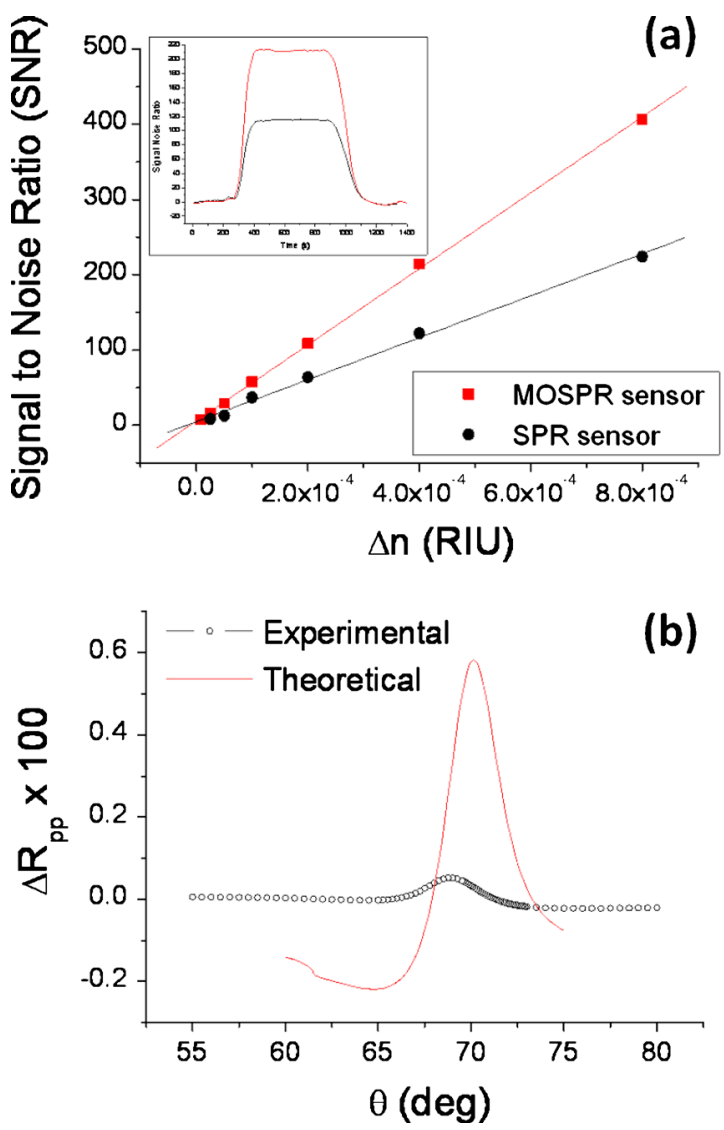

FIG. 5. (Color online) (a) Experimental response of the MOSPR and intensity-interrogated SPR sensors to the same $n_{d}$ variations. The MP transducer is a $30 \mathrm{~nm} \mathrm{Au}_{\text {top }} / 5 \mathrm{~nm} \mathrm{Fe} / 5 \mathrm{~nm} \mathrm{Au}_{\text {down }}$ trilayer and the SPR transducer a Au layer of $48 \mathrm{~nm}$. The inset shows the temporal response of both sensors for the same refractive-index change $\left(\Delta n=2 \times 10^{-4}\right.$ RIU). (b) Theoretical and experimental $\Delta \mathrm{R}_{\mathrm{pp}}$ of the MP transducer employed (30 nm $\mathrm{Au}_{\text {top }} / 5 \mathrm{~nm} \mathrm{Fe} / 5 \mathrm{~nm} \mathrm{Au}_{\text {down }} / 2 \mathrm{~nm} \mathrm{Cr} /$ glass). In the theoretical calculations we used the optical and MO constants determined by Lee. ${ }^{27}$

employed, allowing several cycles of regenerations using $\mathrm{HCl} 0.1 \mathrm{M}$ or formamide solutions after the biosensing interactions.

\section{CONCLUSIONS}

We have analyzed the MP and sensing features of a novel transducer based on a $\mathrm{Au} / \mathrm{Fe} / \mathrm{Au}$ trilayer. Similarly to other MP structures, we observed the possibility to modulate the SPP by an external magnetic field, the amplification of the TMOKE upon SPP excitation and the sharp dependence of this TMOKE enhancement on the SPP resonance condition. Furthermore, we have also confirmed a linear dependence of the $\mathrm{MO}$ modulation factor with respect to the thickness of the thin ferromagnetic layer, along with an inversely proportional dependence of this parameter with respect to the upper $\mathrm{Au}$ layer thickness. Besides, this study has provided valuable information for the future design of highly sensitive sensing transducers. We have confirmed a twofold enhancement of the MOSPR sensor sensitivity with respect to a standard SPR sensor through a direct comparison (i.e., using the same flow system and the same light and detection sources). Nevertheless, the sensitivity enhancement of $\mathrm{Au} / \mathrm{Fe} / \mathrm{Au}$ trilayer is lower than that theoretically expected probably due to interface roughness that reduces the effective $\mathrm{Fe}$ thickness, leading to a smaller MO activity than expected for the nominal $\mathrm{Fe}$ thickness. On the other hand, the $\mathrm{Au} / \mathrm{Fe} / \mathrm{Au}$ structure provides excellent chemical stability and resistance to the oxidation. New and promising alternatives in the development of MP transducers would be structures with sharper interfaces, such as epitaxial structures, or the use of $\mathrm{Fe} / \mathrm{Co}$ alloys as ferromagnetic material, where the Fe would provide stability and solve the lack of resistance of the $\mathrm{Au} / \mathrm{Co} \mathrm{MP}$ systems. In addition, as the MP transducer interface with the external medium is gold, full compatibility with the well-known SPR biofunctionalization protocols can be easily achieved.

\section{ACKNOWLEDGMENTS}

Financial support is acknowledged from: FUNCOAT, MICINN Consolider Ingenio 2010 under Project No. CSD2008-00023; MAGPLAS MICINN under Project No. MAT2008-06765-C02-01/NAN; MICROSERES CAM under Project No. S2009/TIC-1476; and M. Botín Foundation.

David Regatos acknowledges financial support from "Programa de Formación de Investigadores del Departamento de Educación, Universidades e Investigación" of the Gobierno Vasco (Spain). B. Sepulveda acknowledges the financial support from the Ramon y Cajal program from MICINN.

${ }^{1}$ H. Raether, Surface Plasmons on Smooth and Rough Surfaces and on Gratings (Springer-Verlag, Berlin, 1988).

${ }^{2}$ S. A. Maier, Plasmonics (Springer, New York, 2007).

${ }^{3}$ B. Liedberg, C. Nylander, and I. Lundström, Biosens. Bioelectron. 10, i (1995).

${ }^{4}$ J. Homola, Chem. Rev. 108, 462 (2008).

${ }^{5}$ J. Homola, Surface Plasmon Resonance Based Sensors (Springer-Verlag, Berlin, 2006)

${ }^{6}$ M. A. Otte, B. Sepúlveda, W. Ni, J. P. Juste, L. M. Liz-Marzán, and L. M. Lechuga, ACS Nano 4, 349 (2010).

${ }^{7}$ M. Piliarik and J. Homola, Opt. Express 17, 16505 (2009).

${ }^{8}$ I. R. Hooper and J. R. Sambles, Appl. Phys. Lett. 85, 3017 (2004).

${ }^{9}$ S. Patskovsky, M. Maisonneuve, M. Meunier, and A. V. Kabashin, Opt. Express 16, 21305 (2008)

${ }^{10}$ C.-M. Wu, Z.-C. Jian, S.-F. Joe, and L.-B. Chang, Sens. Actuators B 92 , 133 (2003).

${ }^{11}$ P. P. Markowicz, W. C. Law, A. Baev, P. N. Prasad, S. Patskovsky, and A. Kabashin, Opt. Express 15, 1745 (2007).

${ }^{12}$ A. V. Kabashin, S. Patskovsky, and A. N. Grigorenko, Opt. Express 17, 21191 (2009).

${ }^{13}$ B. Sepúlveda, A. Calle, L. M. Lechuga, and G. Armelles, Opt. Lett. 31, 1085 (2006).

${ }^{14}$ B. D. Guenther, Modern Optics (Wiley, New York, 1990).

${ }^{15}$ B. Sepulveda, L. M. Lechuga, and G. Armelles, J. Lightwave Technol. 24, 945 (2006)

${ }^{16}$ J. B. González-Díaz, A. Garcia-Martin, G. Armelles, J. M. Garcia-Martin, C. Clavero, A. Cebollada, R. A. Lukaszew, J. R. Skuza, D. P. Kumah, and R. Clarke, Phys. Rev. B 76, 153402 (2007).

${ }^{17}$ P. E. Ferguson, O. M. Stafsudd, and R. F. Wallis, Physica B \& C 89, 91 (1977).

${ }^{18}$ V. I. Safarov, V. A. Kosobukin, C. Hermann, G. Lampel, J. Peretti, and C. Marlière, Phys. Rev. Lett. 73, 3584-3587 (1994).

${ }^{19}$ C. Hermann, V. A. Kosobukin, G. Lampel, J. Peretti, V. I. Safarov, and P. Bertrand, Phys. Rev. B 64, 235422 (2001).

${ }^{20}$ J. Treviño, A. Calle, J. M. Rodríguez-Frade, M. Mellado, and L. M. Lechuga, Talanta 78, 1011 (2009).

${ }^{21}$ L. Carrascosa, A. Calle, and L. Lechuga, Anal. Bioanal. Chem. 393, 1173 (2009).

${ }^{22}$ D. R. Shankaran, K. V. Gobi, and N. Miura, Sens. Actuators B 121, 158 
(2007).

${ }^{23}$ E. Mauriz, A. Calle, J. Manclús, A. Montoya, and L. Lechuga, Anal. Bioanal. Chem. 387, 1449 (2007).

${ }^{24}$ J. H. Weaver, C. Krafka, D. W. Lynch, and E. E. Koch, Appl. Opt. 20, 1124_1 (1981).
${ }^{25}$ E. Kretshmann, Z. Naturforsch. B 23A, 2135 (1968).

${ }^{26}$ L. Uba, A. Polewko, S. Uba, R. Gontarz, A. N. Yaresko, and V. N. Antonov, Phys. Status Solidi A 196, 145 (2003).

${ }^{27}$ Y. P. Lee, Y. V. Kudryavtsev, V. V. Nemoshkalenko, R. Gontarz, and J. Y. Rhee, Phys. Rev. B 67, 104424 (2003). 